\title{
Binding Stability of $\beta-C D$ on MWCNTS: Role of Washing Cycle on the $\beta$-CD Coating
}

\author{
Zeinab Abbas Jawad, ${ }^{1 *}$ Abdul Latif Ahmad, ${ }^{2}$ Siew Chun Low, ${ }^{2}$ Eric Wong Tung Seng, ${ }^{1}$ \\ Chek Fong Bryan Soh, ${ }^{1}$ Lavania Sugu ${ }^{1}$ and Thiam Leng Chew ${ }^{3}$ \\ ${ }^{1}$ Department of Chemical Engineering, School of Engineering and Science, \\ Curtin University Sarawak Malaysia, CDT 250, 98009 Miri, Sarawak, Malaysia \\ ${ }^{2}$ School of Chemical Engineering, Engineering Campus, \\ Universiti Sains Malaysia, 14300 Nibong Tebal, Penang, Malaysia \\ ${ }^{3}$ Department of Chemical Engineering, Faculty of Engineering, Universiti Teknologi \\ PETRONAS, 32610 Bandar Seri Iskandar, Perak, Malaysia
}

*Corresponding author: zeinab.aj@curtin.edu.my

Published online: 15 February 2017

To cite this article: Jawad, Z. A. et al. (2017). Binding stability of $\beta$-CD on MWCNTS: Role of washing cycle on the $\beta$-CD coating. J. Phys. Sci., 28(Supp. 1), 145-153, https://doi.org/10.21315/jps2017.28.s1.9

To link to this article: https://doi.org/10.21315/jps2017.28.s1.9

\begin{abstract}
The functionalisation of carbon nanotubes (CNTS) represents a strategy not only to improve the CNTs dispersion, solubility, and processability, but also to allow strong interfacial interactions to take place between CNTs and guest molecules. In this study, beta-cyclodextrins ( $\beta$-CD) was used to functionalise the sidewalls of multi-walled CNTs (MWCNTs) for better stability. The role of washing cycles on the $\beta$-CD binding was investigated. The results indicated that the mean diameter of MWCNTs was retained at almost the same size even after three cycles of washing. This observation confirmed the strong binding between MWCNTs and $\beta-C D$.
\end{abstract}

Keywords: $\beta$-cyclodextin, multi-walled carbon nanotubes, non-covalent functionalisation, washing cycles, binding stability

\section{INTRODUCTION}

Carbon nanotubes (CNTs) are inorganic disperse phases that differ substantially from other molecular sieves. ${ }^{1}$ This is because of its synthesised method from metal oxides which is also considered as the inorganic materials as well as its undissolved behaviour in the organic solvents. The CNTs are a member of the 
fullerene structural family, which consist of six-member carbon rings (hexagon) in a honeycomb lattice relative to the axis of the nanotubes. ${ }^{2}$ CNTs are different from others carbon materials such as graphite, diamond and fullerene owing to its onedimensional carbon with an aspect ratio greater than $1000 .^{3}$

The functionalised CNTs may exhibit enhancement in mechanical or electrical properties as compared to pristine CNTs. ${ }^{4}$ In this regard, the CNTs functionalisation is an attractive target for many scientists. Based on the principles of CNTs functionalisation, it can be classified into two approaches: covalent and noncovalent functionalisation. ${ }^{5}$

In this study, the non-covalent functionalisation was applied. It is defined as the electrostatic binding between the CNTs and molecules. It can maintain the activity between CNTs and guest molecules successfully. ${ }^{5}$ The main advantages of the non-covalent functionalisation method are its low cost, commercially available material, fast, easy to carry out, and simple up-scalability to pilot plant level with maintained structural integrity of CNTs. ${ }^{6}$ The non-covalent functionalisation can be classified based on the interaction between CNTs and guest molecules into surfactant adsorption, endohedral method, and polymer wrapping. ${ }^{4}$

In case of suspension of the CNTs in the presence of polymers, this will result in wrapping of the polymer around the CNTs forming supermolecular complexes of CNTs. Polymer wrapping is a typical example of non-covalent functionalisation that can be achieved through van der Waals interactions and $\pi$ - $\pi$ stacking between the CNTs and the polymer chains. ${ }^{4}$ Based on Panhuis et al., the polymer backbone had provided a strong binding to the CNTs $(123 \mathrm{~kJ} / \mathrm{mol})$ compared to the side chains $(36 \mathrm{~kJ} / \mathrm{mol}) .^{7}$ Furthermore, they found it energetically more favourable for the polymer to lie along the parallel axis instead of mapping onto the chirality of the underlying tube. ${ }^{7}$

Ma et al. showed that the dispersion stability of functionalised-CNTs was better compared to pristine CNTs. ${ }^{8}$ This was due to the improvement in the abilities of the hydrogen-bonding on the CNTs surface and the CNTs surface was converted from the hydrophobic to the hydrophilic state. ${ }^{8}$

Based on Jiang et al., the best stability, accessibility, and selectivity can be achieved through covalent bonding because of its capability to control the location of the biomolecules, improved stability, accessibility, selectivity, and reduced leaching. ${ }^{9}$ Realising the important of the non-covalent functionalisation of CNTs, the aim of the present work is to investigate the binding stability of the non-covalent functionalised MWCNTs that was prepared via Chen's soft cutting method. 
Here, a study was conducted to evaluate the effect of washing cycle on the binding of beta-cyclodextrins $(\beta-C D)$.

\section{EXPERIMENTAL}

\subsection{Materials}

Materials used in this study were listed in Table 1. Also, the transmission electron microscope (TEM) micrograph of Pristine-MWCNTs (MWCNTs-P) is shown in Figure 1.

Table 1: List of materials and chemicals used.

\begin{tabular}{lcc}
\hline Chemical & Assay & Supplier \\
\hline Multi-walled carbon nanotubes & $>95 \%$ (lengths ranging & Shenzhen Nanotech Port \\
(MWCNTs) & from 5 to $50 \mu \mathrm{m})$ & Co. Ltd, China \\
Beta-cyclodextrin $(\beta-\mathrm{CD})$ & $98.3 \%$ & Merck, Malaysia \\
Ethanol & $>99 \%$ & Merck, Malaysia \\
\hline
\end{tabular}

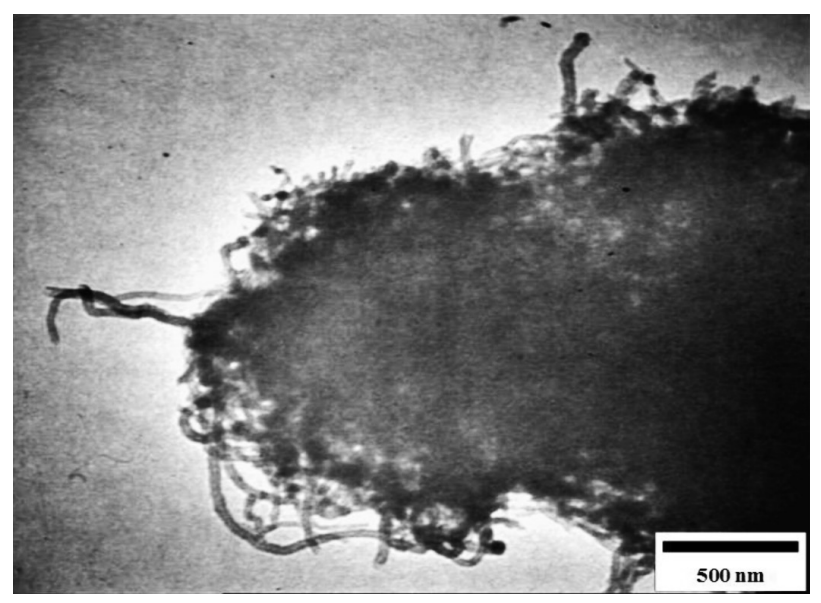

Figure 1: TEM micrograph of MWCNTs-P.

\subsection{Stability of the Functionalised Mwents}

The MWCNTs was functionalised according to previously published works. ${ }^{10-13}$ Then, the functionalised MWCNTs was washed for first, second and third time using deionised water in order to remove the excess amount of $\beta$-CD. The 
suspension solution was filtered using filter paper (Hangzhou Xinhua, China) with pore size of $0.2 \mu \mathrm{m}$. After that, the functionalised MWCNTs was dried before it further characterised using TEM. ${ }^{14}$

\subsection{Characterisation}

The structures and properties of the MWCNTs were obtained from the TEM Philips CM12 equipped with DOCU version 3.2 image analysis systems. Prior to TEM analysis, samples were sonicated in chloroform for $10 \mathrm{~min}$ and then dropped onto $\mathrm{Cu}$ grid of TEM holder. At least three samples were analysed to confirm the structure of MWCNTs.

The diameter distribution of the samples was measured from TEM images. It was based on the frequency count of MWCNT's diameters that were observed in these images. Approximately 40-90 measurements were taken. The average diameters and the distribution trends together with the standard errors were tabulated.

\section{RESULTS AND DISCUSSIONS}

In order to investigate whether the wrapping of $\beta-\mathrm{CD}$ around MWCNTs was still maintained after a few repeated washing steps, the stability of functionalised MWCNTs was determined. In Figure 2, the functionalised MWCNTs were depicted to have a uniform coating layer of $\beta-C D$.

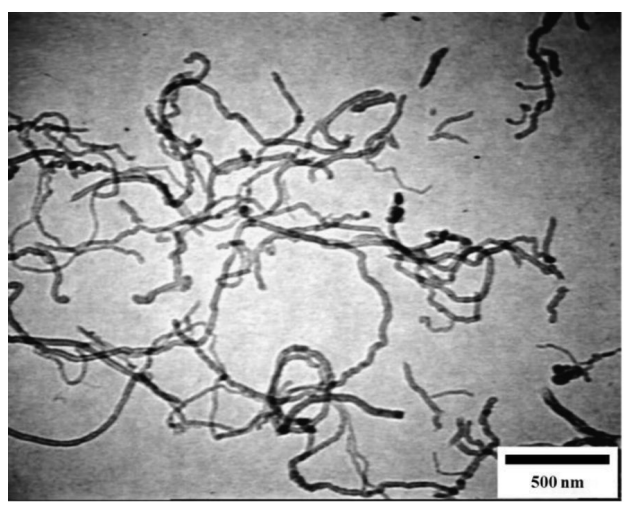

(a)

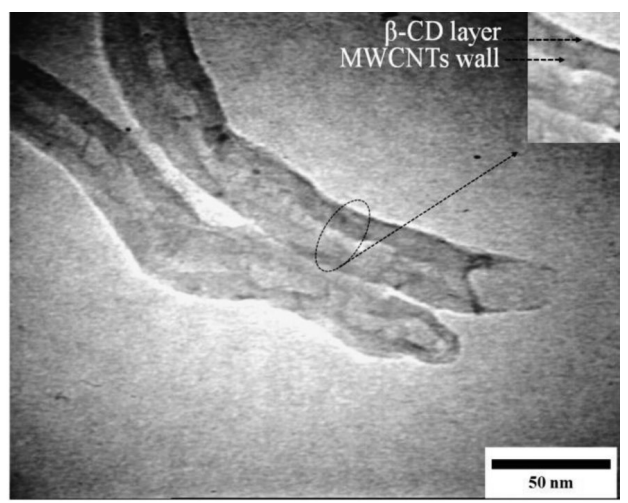

(b)

Figure 2: TEM micrographs of functionalised MWCNTs without washing at (a) $500 \mathrm{~nm}$ and (b) $50 \mathrm{~nm}$. 
In addition, it was found that the functionalised MWCNTs without carrying out any washing steps had a mean outer diameter of $33.75 \pm 1.16 \mathrm{~nm}$, as shown in Figure 3.

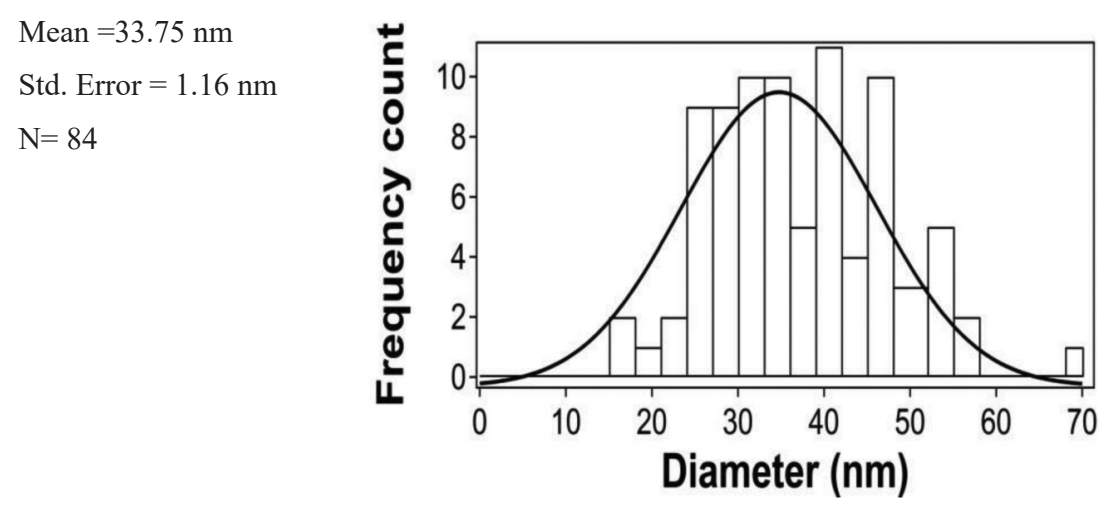

Figure 3: Diameter distribution for functionalised MWCNTs without washing.

After three washing cycles, the functionalised MWCNTs were shown to have retained a uniform coating structure (Figure 4(a)-(f)). Thus, this confirmed the strong binding between MWCNTs and $\beta-C D$ although being washed. This finding is important because it confirmed the successful functionalisation of MWCNTs using $\beta-C D$ with a strong and stable structure. This was due to the $\beta-C D$ was wrapped strongly around the CNTs through van der Waals interactions and $\pi-\pi$ stacking between the CNTs and the polymer chains. ${ }^{4}$

In Figure 5, the diameter distributions of functionalised MWCNTs obtained after first, second, and third washing cycles were compared. For the first washing cycle, it was found that the functionalised MWCNTs had a mean outer diameter of 32.46 $\pm 1.35 \mathrm{~nm}$ (Figure 5(a)). By carrying out the washing process repeatedly on the functionalised MWCNTs for the second and third cycle, the mean diameter of MWCNTs was retained at almost the same sizes at $31.71 \pm 0.76 \mathrm{~nm}$ and $31.61 \pm$ $1.02 \mathrm{~nm}$ (Figure 5(b)-(c)), respectively. This proved the firm binding of $\beta$-CD to the MWCNTs. Eventually, the findings of the present work were in line with the literature of non-covalent functionalisation. ${ }^{15}$ Where, the wrapping of variety of polymers along the CNTs sides is robust, uniformly distributed and is not depend on the presence of excess polymer in solution. ${ }^{15}$ 


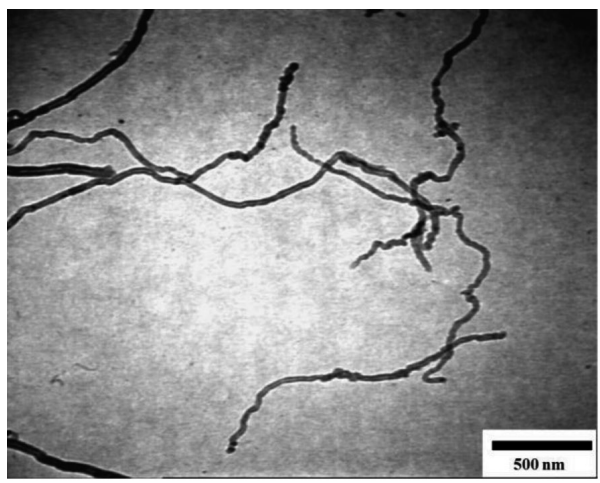

(a)

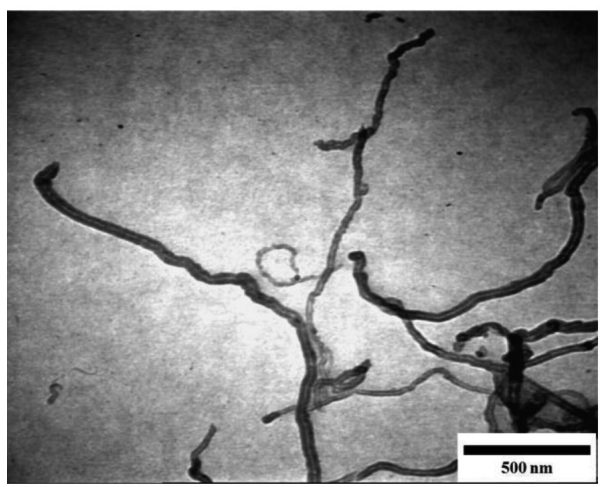

(c)

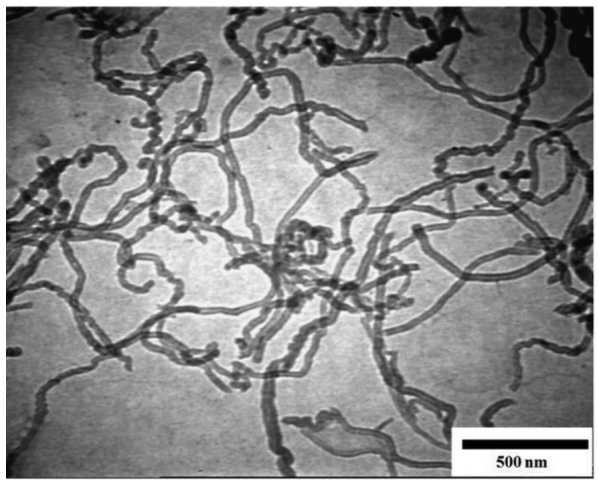

(e)

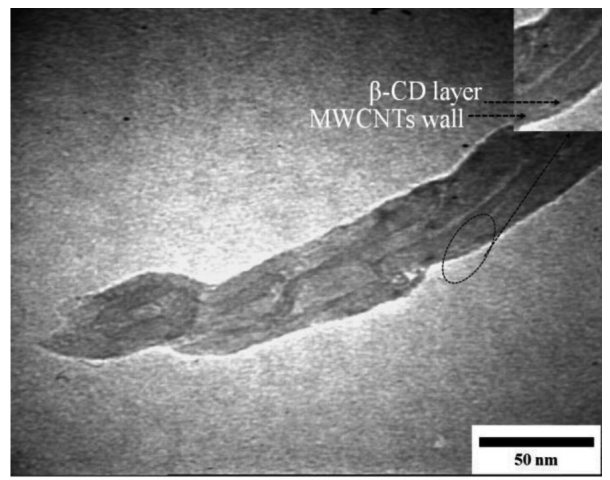

(b)

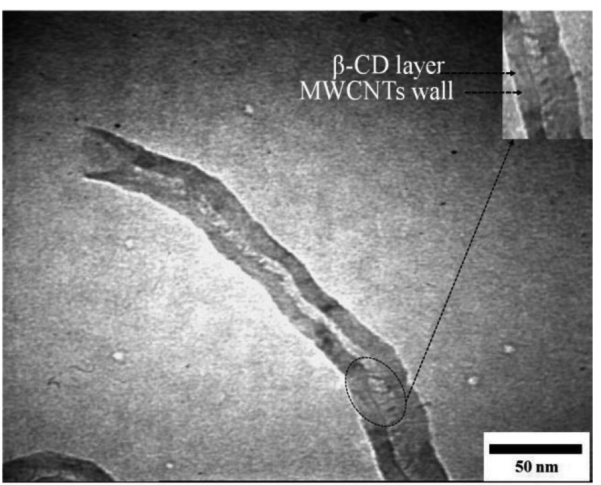

(d)

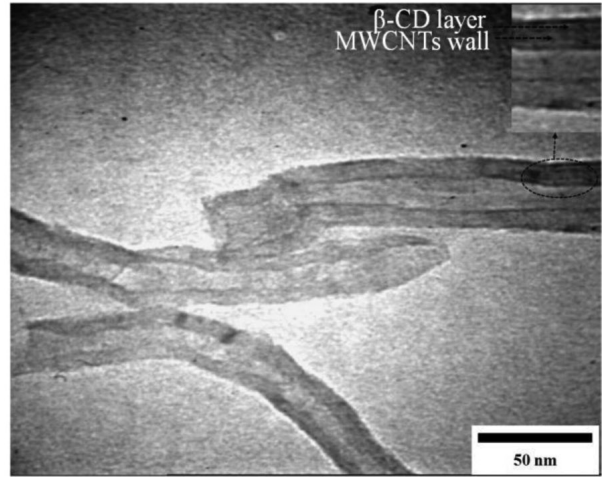

(f)

Figure 4: TEM micrographs of MWCNTs after $(a, b)$ first, $(c, d)$ second, and $(e, f)$ third washing cycle. 


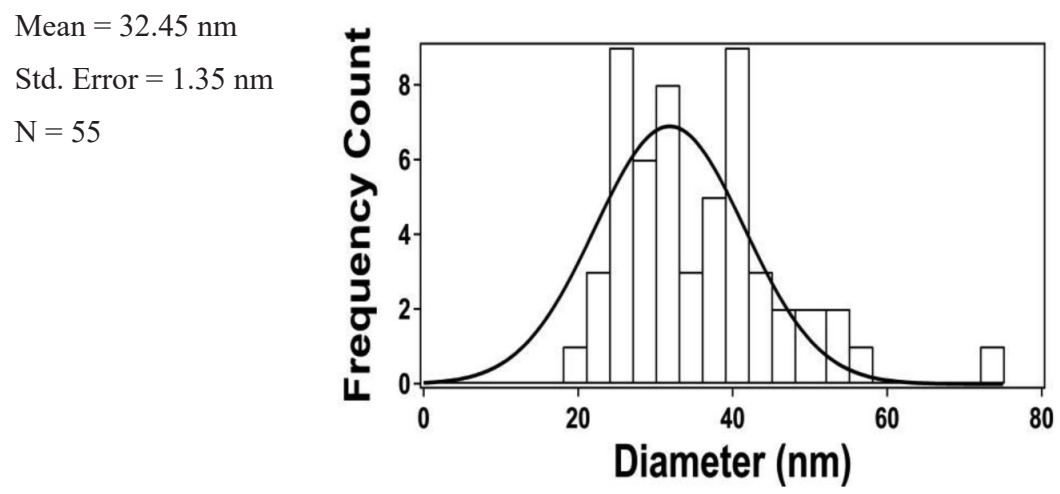

(a)

Mean $=31.71 \mathrm{~nm}$

Std. Error $=0.76 \mathrm{~nm}$ $\mathrm{N}=40$

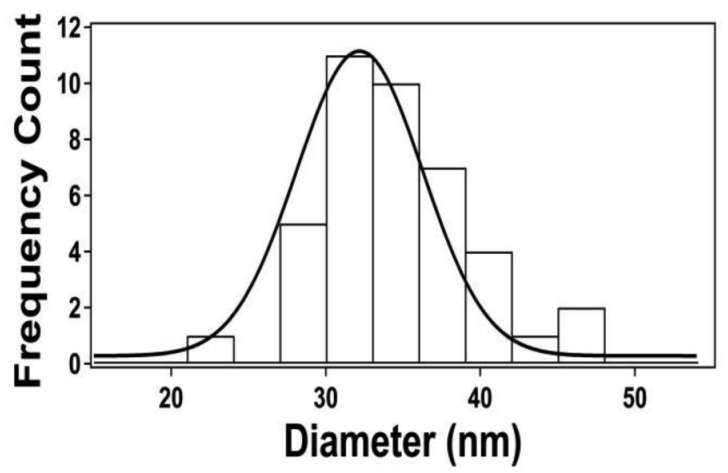

(b)

Mean $=31.61 \mathrm{~nm}$

Std. Error $=1.02 \mathrm{~nm}$ $\mathrm{N}=80$

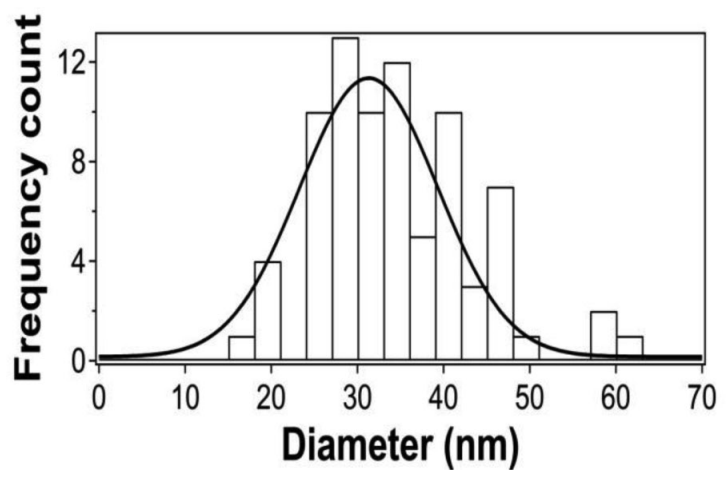

(c)

Figure 5: Diameter distribution for functionalised MWCNTs at (a) first, (b) second, and (c) third washing cycle. 


\section{CONCLUSION}

In the present work, the MWCNTs were successfully functionalised using $\beta$-CD through Chen's soft cutting technique. The good binding stability of $\beta-C D$ was demonstrated via repeated washing cycles. The TEM results indicated that the functionalisation of MWCNTs had retained a uniform coating. This finding is important because it confirmed the successful functionalisation of MWCNTs using $\beta-C D$ with a strong and stable structure. This strong binding of $\beta-C D$ around the CNTs is very important to maintain the well dispersed MWCNTs within the polymer matrix of the mixed matrix membrane and enhance its separation performance later. In this case, there is no possibility to detach the $\beta$-CD from the MWCNTs due to its strong wrapping around the CNTs through van der Waals interactions and $\pi-\pi$ stacking between the CNTs and the polymer chains.

\section{ACKNOWLEDGEMENTS}

The authors thank the Ministry of Higher Education (MOHE) Malaysia for providing Fundamental Research Grant Scheme (FRGS) (MOHE Ref. No. is FRGS/1/2015/TK02/CURTIN/03/1) and Cost Center: 001048. Also, the authors would like to thank LRGS USM (Account No: 304/PJKIMIA/6050296/U124), and Curtin Cost Center 001047.

\section{REFERENCES}

1. Ismail, A. F. et al. (2009). Transport and separation properties of carbon nanotube-mixed matrix membrane. Sep. Purif. Technol., 70, 12-26, http://dx.doi.org/10.1016/j.seppur.2009.09.002.

2. Ismail, A. F. et al. (2011). Gas separation performance of polyethersulfone/ multi-walled carbon nanotubes mixed matrix membranes. Sep. Purif. Technol., 80, 20-31, http://dx.doi.org/10.1016/j.seppur.2011.03.031.

3. Kim, J. K. (2011). Introduction. In (Ed.) Kim, J. K. Functionalization of CNTs. Florida: CRC Press.

4. Kim, J. K. (2011). Functionalization of CNTs. Florida: CRC Press.

5. Xue, Y. et al. (2011). Noncovalent functionalization of carbon nanotubes with lectin for label-free dynamic monitoring of cell-surface glycan expression. Anal. Biochem., 410, 92-97, https://dx.doi.org/10.1016/j.ab.2010.11.019. 
6. Prado, L. d. A. et al. (2009). Noncovalent functionalization of multiwalled and double-walled carbon nanotubes: Positive effect of the filler functionalization on high glass transition temperature epoxy resins. Polym. Sci. B Polym. Phys., 47, 1860-1868, https://doi.org/10.1002/polb.21789.

7. Panhuis, M. et al. (2003). Selective interaction in a polymer-single-wall carbon nanotube composite. J. Phys. Chem. B, 107, 478-482, https://doi.org/ 10.1021/jp026470s.

8. Ma, P. C., Kim, J.-K. \& Tang, B. Z. (2006). Functionalization of carbon nanotubes using a silane coupling agent. Carbon, 44, 3232-3238, https://doi.org/10.1016/j.carbon.2006.06.032.

9. Jiang, K. et al. (2004). Protein immobilization on carbon nanotubes via a two-step process of diimide-activated amidation. J. Mater. Chem., 14, 37-39, https://doi.org/10.1039/B310359E.

10. Jawad, Z. A. et al. (2015). Incorporation of inorganic carbon nanotubes fillers into the CA polymeric matrix for improvement in $\mathrm{CO} 2 / \mathrm{N} 2$ separation. Curr. Nanosci., 11, 69-79.

11. Jawad, Z. A. et al. (2015). Influence of solvent exchange time on mixed matrix membrane separation performance for $\mathrm{CO} 2 / \mathrm{N} 2$ and a kinetic sorption study. J. Membr. Sci., 476, 590-601, https://doi.org/10.1016/j. memsci.2014.11.008.

12. Ahmad, A. L. et al. (2013). A cellulose acetate/multi-walled carbon nanotube mixed matrix membrane for CO2/N2 separation. J. Membr. Sci., 451, 55-66, https://dx.doi.org/10.1016/j.memsci.2013.09.043.

13. Ahmad,A.L. etal.(2013). The functionalization of beta-cyclodextrins on multi walled carbon nanotubes: Effects of the dispersant and non aqueous media. Curr. Nanosci., 9, 93-102, https://doi.org/10.2174/157341313805117839.

14. Chen, J., Dyer, M. J. \& Yu, M. F. (2001). Cyclodextrin-mediated soft cutting of single-walled carbon nanotubes. J. Am. Chem. Soc., 123, 6201-6202, https://doi.org/10.1021/ja015766t.

15. O'Connell, M. J. et al. (2001). Reversible water-solubilization of singlewalled carbon nanotubes by polymer wrapping. Chem. Phys. Lett., 342, 265-271, https://doi.org/10.1016/S0009-2614(01)00490-0. 
